\title{
Clinical Outcomes of Pediatric COVID-19 During Two Waves of Different Variants Circulation in Latin America
}

\author{
Adriana Yock-Corrales ${ }^{1}$ (1) $\cdot$ Jessica Gomez-Vargas ${ }^{1} \cdot$ Rolando Ulloa-Gutierrez $^{2}$ (D) Martin Brizuela $^{3}$. \\ Olguita del Aguila ${ }^{4} \cdot$ Verónica Kozicki $^{5}$. Jacopo Lenzi ${ }^{5} \cdot$ Danilo Buonsenso $^{6}$
}

Received: 14 October 2021 / Accepted: 24 December 2021 / Published online: 4 March 2022

(c) Dr. K C Chaudhuri Foundation 2022

To the Editor: The clinical impact of COVID-19 has been milder on children compared with adults [1]. Latin America is characterized by a specific geopolitical situation and data are necessary to understand the real impact on children, and allocate resources to face the local situation of the COVID19 pandemic. We conducted a prospective study of clinical characteristics and outcomes of children $<17 \mathrm{y}$ with a positive SARS-CoV-2 test from March 1, 2020 through June 30, 2021 in three referral hospitals during two pandemic waves. A total of 992 children were included, with a median age of 5.3 y (IQR 0.6-9). In 59.7\% of the patients, the index case was unknown. Thirty-two percent needed hospital admission and the main symptoms were fever (80.4\%), upper respiratory tract symptoms (45\%), and diarrhea and/ or vomiting (39.9\%). Seventy-nine (8\%) patients were diagnosed with MIS-C and 53 (5.3\%) required intensive care unit (ICU) admission. Mortality, need for respiratory support, and ICU hospitalization did not significantly change. During the second wave, fewer children had a known source of infection and fewer household cases $(p<0.0001)$. Clinical

Adriana Yock-Corrales

adriyock@gmail.com

1 Pediatric Emergency Department, Hospital Nacional de Niños "Dr Carlos Sáenz Herrera", CCSS, San José 957-1100, Costa Rica

2 Infectious Disease Division, Hospital Nacional de Niños “Dr Carlos Sáenz Herrera", CCSS, San José, Costa Rica

3 Pediatric Infectious Disease, Hospital Isidoro Iriarte, Quilmes, Buenos Aires, Argentina

4 Unidad de Infectología Pediátrica del Hospital Nacional Edgardo Rebagliati Martins, Lima, Perú

5 Department of Biomedical and Neuromotor Sciences, Alma Mater Studiorum - University of Bologna, Bologna, Italy

6 Department of Woman and Child Health and Public Health, Fondazione Policlinico Universitario A. Gemelli and Global Health Research Institute, Istituto Di Igiene, Università Cattolica del Sacro Cuore, Roma, Italia presentations changed, with more children presenting with lower respiratory tract symptoms and diarrhea $(p<0.0001)$.

This is the largest multinational study comparing COVID19 presentation in children during two different pandemic waves. Despite debates and fear of possibly more serious diseases in children, our study found that ICU hospitalization and mortality did not change. Interestingly, we found a change in clinical presentation with more children having symptoms of LRTI and diarrhea. Also, fewer children had known SARS-CoV-2 cases within households. Fear of more severe disease could influence decisions about school closures, with a well-known impact on global health [2]. Although this study includes patients before the emergence of the delta variant, it provides the first evidence of similar disease severity in children in Latin America.

Data Availability On request.

Code Availability The code and data used to produce the results and analyses presented in this paper will be available upon request.

\section{Declarations}

Ethics Approval The study was approved by each institution.

Consent for Publication Consent for publication of this data was obtained from the IRB. CENDEISSS- AB- 1990- 2020.

Conflict of Interest None.

\section{References}

1. Kara AA, Böncüoğlu E, Kiymet E, et al. Evaluation of predictors of severe-moderate COVID-19 infections at children: a review of 292 children. J Med Virol. 2021;93:6634-40.

2. Buonsenso D, Roland D, De Rose C, et al. Schools closures during the COVID-19 pandemic: A catastrophic global situation. Pediatr Infect Dis J. 2021;40:e146-50.

Publisher's Note Springer Nature remains neutral with regard to jurisdictional claims in published maps and institutional affiliations. 